\title{
Characterization of the Besov Spaces via the Commutator Operator of Coifman, Rochberg and Weiss
}

\section{PALUSZYŃSKI}

Abstract. Let $\mathrm{C}_{f}(g)=T(f \cdot g)-f \cdot T(g)$ where $T$ is the Calderón-Zygmund singular integral operator. It is a well known result of Coifman, Rochberg and Weiss that the boundedness of this operator on $L^{p}\left(\mathbf{R}^{n}\right)$ is equivalent to $f$ being in the space BMO. In this paper we introduce a version of this operator which allows $f$ to be in the Besov-Lipschitz class $\dot{\Lambda}_{\beta}$, with $0<\beta<n$.

0. Introduction. A well known result of Coifman, Rochberg and Weiss $([2],[6],[14])$ states that the commutator operator

$$
\mathrm{C}_{f}(g)=T(f \cdot g)-f \cdot T(g)
$$

(where $T$ is a Calderón-Zygmund singular integral operator) is bounded on some $L^{p}\left(\mathbf{R}^{n}\right), 1<p<\infty$, if and only if $f \in$ BMO. There are other links between the boundedness properties of the operator $\mathrm{C}_{f}$ and the smoothness of $f$. A particular case of the result of Janson ([6]) states that $\mathrm{C}_{f}: L^{p}\left(\mathbf{R}^{n}\right) \rightarrow L^{q}\left(\mathbf{R}^{n}\right)$ is bounded, $1<p<q<\infty$, if and only if $f \in \operatorname{Lip} \beta, \beta=n(1 / p-1 / q)$. Here, $\operatorname{Lip} \beta$ is the homogeneous Lipschitz space determined by the first difference operator. In [14] Uchiyama showed that $\mathrm{C}_{f}$ is a compact operator on some $L^{p}\left(\mathbf{R}^{n}\right)$ if and only if $f \in \mathrm{CMO}$, a subclass of BMO.

A related type of commutator was introduced by Chanillo ([1]). Let $I^{\alpha}$ be the Riesz potential of order $\alpha$, and define

$$
\mathrm{C}_{f}^{\alpha}(g)=I^{\alpha}(f \cdot g)-f \cdot I^{\alpha}(g) .
$$

The result in [1] states that $\mathrm{C}_{f}^{\alpha}: L^{p}\left(\mathbf{R}^{n}\right) \rightarrow L^{q}\left(\mathbf{R}^{n}\right)$ is bounded, $1<p<q<\infty$, $1 / p-1 / q=\alpha / n$, if and only if $f \in$ BMO. For technical reasons, the "only if" part of the proof in [1] is only valid for $\alpha$ an even integer. 
In this paper, we extend and generalize the results from [1] and [6]. Namely, if $1<p<\infty, 0<\beta<1$, then $f \in \dot{\Lambda}_{\beta}$ if and only if $\mathrm{C}_{f}: L^{p}\left(\mathbf{R}^{n}\right) \rightarrow \dot{F}_{p}^{\beta, \infty}$ is bounded. Here, $\dot{\Lambda}_{\beta}$ is the homogeneous Lipschitz space, and $\dot{F}_{p}^{\beta, \infty}$ is the homogeneous Triebel-Lizorkin space. The corresponding result is true for $\mathrm{C}_{f}^{\alpha}$. If $1<p<q<\infty, 0<\beta<1,1 / p-1 / q=\alpha / n$, then $f \in \dot{\Lambda}_{\beta}$ if and only if $\mathrm{C}_{f}^{\alpha}: L^{p}\left(\mathbf{R}^{n}\right) \rightarrow \dot{F}_{q}^{\beta, \infty}$ is bounded. If $p<n(\alpha+\beta)$, then $f \in \dot{\Lambda}_{\beta}$ if and only if $\mathrm{C}_{f}^{\alpha}: L^{p}\left(\mathbf{R}^{n}\right) \rightarrow L^{r}\left(\mathbf{R}^{n}\right)$ is bounded, $1 / p-1 / r=(\alpha+\beta) / n$. We note that the methods we employ give a complete proof of the result of Chanillo in [1]. In Section 2 we give a definition of the generalized commutator operators $\mathrm{C}_{f, k}$ and $\mathrm{C}_{f, k}^{\alpha}$. The result in [6] implies that if $n(1 / p-1 / q)>1$, then $\mathrm{C}_{f}$ : $L^{p}\left(\mathbf{R}^{n}\right) \rightarrow L^{q}\left(\mathbf{R}^{n}\right)$ is bounded if and only if $f$ is a constant. In this case, the characterization of the spaces $\dot{\Lambda}_{\beta}$ can be obtained using the operators $\mathrm{C}_{f, k}$ and $\mathrm{C}_{f, k}^{\alpha}, k>n(1 / p-1 / q)$. Let $K(x)$ be the Calderón-Zygmund kernel, $K^{\alpha}(x)=$ $\omega_{\alpha}|x|^{\alpha-n}$. We then define

$$
\begin{gathered}
\mathrm{C}_{f, k} g(x)=\int_{\mathbf{R}^{n}} \Delta_{h}^{k} f(x) K(h) g(x+h) d h, \\
\mathrm{C}_{f, k}^{\alpha} g(x)=\int_{\mathbf{R}^{n}} \Delta_{h}^{k} f(x) K^{\alpha}(h) g(x+h) d h,
\end{gathered}
$$

where $\Delta_{h}^{k}$ is the $k^{\text {th }}$ order difference operator with step size $h$. If $k=1$, these operators coincide with the original commutator operators. We obtain the following characterization. Let $1<p<q<\infty, 1 / p-1 / q=\beta / n, 0<\beta<k, k$ an integer. Then, $\mathrm{C}_{f, k}: L^{p}\left(\mathbf{R}^{n}\right) \rightarrow L^{q}\left(\mathbf{R}^{n}\right)$ is bounded if and only if $f=f_{1}+P$, with $f_{1} \in \dot{\Lambda}_{\beta}$ and $P$ is a polynomial of degree less than $k$. The same is true for $\mathrm{C}_{f, k}^{\alpha}$ provided $1 / p-1 / q=(\alpha+\beta) / n$.

We denote by $\Delta_{h}^{k}$ the $k^{\text {th }}$ difference operator. That is,

$$
\begin{aligned}
\Delta_{h}^{1} f(x) & =\Delta_{h} f(x)=f(x+h)-f(x), \\
\Delta_{h}^{k+1} f(x) & =\Delta_{h}^{k} f(x+h)-\Delta_{h}^{k} f(x),
\end{aligned}
$$$$
k \geq 1
$$

$Q(x, t)$ denotes a cube centered at $x$, with side length $t$, sides parallel to the axes. For $c>0, c Q$ denotes the cube with the same center as $Q$, and the side length increased $c$ times. We denote by $x_{Q}$ the center of $Q$. For a cube $Q$ we let

$$
f_{Q}=\frac{1}{|Q|} \int_{Q} f .
$$

For $1<p<\infty, p^{\prime}$ is the dual, $1 / p+1 / p^{\prime}=1$, and we use the notation

$$
\|f\|_{p}=\|f\|_{L^{p}\left(\mathbf{R}^{n}\right)},
$$


the usual norm in the Lebesgue space $L^{p}\left(\mathbf{R}^{n}\right)$. For $\beta>0$, the Lipschitz space $\dot{\Lambda}_{\beta}$ is the space of functions $f$ such that

$$
\|f\|_{\dot{\Lambda}_{\beta}}=\sup _{\substack{x, h \in \mathbf{R}^{n} \\ h \neq 0}} \frac{\left|\Delta_{h}^{[\beta]+1} f(x)\right|}{|h|^{\beta}}<\infty .
$$

In this paper a Calderón-Zygmund singular integral kernel is a function $K$ on $\mathbf{R}^{n}$ with the following properties:

$$
K(x)=\frac{\Omega(x)}{|x|^{n}}, \quad \int_{\Sigma_{n-1}} \Omega=0, \quad \Omega \in \mathrm{C}^{\infty}\left(\Sigma_{n-1}\right),
$$

where $\Omega$ is homogeneous of degree 0 ; that is,

$$
\Omega(r x)=\Omega(x) \text { for } r>0,
$$

and $\Sigma_{n-1}$ denotes the unit sphere in $\mathbf{R}^{n}$. The Calderón-Zygmund singular integral operator is the operator $T$, given by the formula

$$
T f(x)=\text { p.v. } \int_{\mathbf{R}^{n}} K(x-y) f(y) d y .
$$

The Riesz potential operator of the order $\alpha, 0<\alpha<n$, denoted by $I^{\alpha}$ is the convolution operator with the kernel $\omega_{\alpha}|x|^{\alpha-n}$ where the constant $\omega_{\alpha}$ is such that

$$
I^{\alpha} I^{\beta}=I^{\alpha+\beta}, \quad \text { provided } \alpha+\beta<n
$$

(see [12]). $M(g)$ denotes the Hardy-Littlewood maximal function, and $M_{r}(g)=$ $\left(M\left(|g|^{r}\right)\right)^{1 / r}$.

Results presented in this paper are part of the author's Ph.D. thesis from Washington University ([9]). The author would like to express his gratitude to his advisors, Professors Mitch Taibleson and Guido Weiss for their assistance. In particular, the idea of studying the Triebel-Lizorkin spaces in the context of the boundedness properties of the commutator operator is due to Professor Weiss.

1. Boundedness properties of the commutator operator. Let $K(x)=$ $\Omega(x) /|x|^{n}$ be a Calderón-Zygmund singular integral kernel, and let $T g=K * g$ be the corresponding Calderón-Zygmund singular integral operator. Let $f \in$ $L_{\text {loc }}^{1} \cap \mathcal{S}^{\prime}$, and let

$$
\begin{aligned}
\mathrm{C}_{f} g(x) & =T(f \cdot g)(x)-f(x) T g(x), \\
\mathrm{C}_{f}^{\alpha} g(x) & =I^{\alpha}(f \cdot g)(x)-f(x) \cdot I^{\alpha} g(x) .
\end{aligned}
$$

We have the following two theorems: 
Theorem 1.1 ([2], [6], [14]). Let $1<p<\infty$. Then, $f \in \mathrm{BMO}$ if and only if $\mathrm{C}_{f}$ is a bounded operator on $L^{p}\left(\mathbf{R}^{n}\right)$.

Theorem $1.2([1])$. Let $1<p<q<\infty,(1 / p)-(1 / q)=\alpha / n$. Then, $f \in \mathrm{BMO}$ if and only if $\mathrm{C}_{f}^{\alpha}$ is a bounded operator from $L^{p}\left(\mathbf{R}^{n}\right)$ to $L^{q}\left(\mathbf{R}^{n}\right)$.

The proof of the "if" part of this theorem given in [1] is only valid for $\alpha$ an even integer. Later we will indicate how we can prove it in full generality.

In this section we will prove the following two results.

Theorem 1.3. Let $1<p<\infty, 0<\beta<1$. Then, the following conditions are equivalent:

(a) $f \in \dot{\Lambda}_{\beta}$,

(b) $\mathrm{C}_{f}$ is a bounded operator from $L^{p}\left(\mathbf{R}^{n}\right)$ to $\dot{F}_{p}^{\beta, \infty}$,

(c) $\mathrm{C}_{f}$ is a bounded operator from $L^{p}\left(\mathbf{R}^{n}\right)$ to $L^{q}\left(\mathbf{R}^{n}\right), 1 / p-1 / q=\beta / n$, if $1 / p>\beta / n$.

Remark. The equivalence of (a) and (c) was proved in [6].

Theorem 1.4. Let $1<p<q<\infty, 0<\beta<1,1 / p-1 / q=\alpha / n$. Then, the following conditions are equivalent:

(a) $f \in \dot{\Lambda}_{\beta}$,

(b) $\mathrm{C}_{f}^{\alpha}$ is a bounded operator from $L^{p}\left(\mathbf{R}^{n}\right)$ to $\dot{F}_{q}^{\beta, \infty}$,

(c) $\mathrm{C}_{f}^{\alpha}$ is a bounded operator from $L^{p}\left(\mathbf{R}^{n}\right)$ to $L^{r}\left(\mathbf{R}^{n}\right), 1 / p-1 / r=(\alpha+\beta) / n$, if $1 / p>(\alpha+\beta) / n$.

In the four theorems above, the norm of $f$, in the appropriate function space, is comparable to the operator norm of $\mathrm{C}_{f}$ and $\mathrm{C}_{f}^{\alpha}$ respectively.

Remark. It is easy to see that in Theorems 1.1 and 1.2 the condition $p>1$ is essential, even if we replace $L^{1}$ by $H^{1}$ on the domain side. In other words, it is not true that $f \in \mathrm{BMO}$ implies that $\mathrm{C}_{f}: H^{1} \rightarrow L^{1}$ is bounded or that $\mathrm{C}_{f}^{\alpha}: H^{1} \rightarrow L^{q}$ is bounded, with $1-1 / q=\alpha / n$.

We now proceed with proofs of Theorems 1.3 and 1.4.

Proof of Theorem 1.3. (a) $\Rightarrow$ (b): Assume $f \in \dot{\Lambda}_{\beta}$. This proof follows the ideas of J.-O. Strömberg, as published in [6]. We will use the following characterizations of relevant function spaces: 


\section{Lemma 1.5.}

(a) For $0<\beta<1,1 \leq q \leq \infty$, we have:

$$
\|f\|_{\dot{\Lambda}_{\beta}} \approx \sup _{Q} \frac{1}{|Q|^{1+\beta / n}} \int_{Q}\left|f-f_{Q}\right| \approx \sup _{Q} \frac{1}{|Q|^{\beta / n}}\left(\frac{1}{|Q|} \int_{Q}\left|f-f_{Q}\right|^{q}\right)^{1 / q}
$$

for $q=\infty$ the formula should be interpreted appropriately.

(b) For $0<\beta<1,1<p<\infty$, we have:

$$
\|h\|_{\dot{F}_{p}^{\beta, \infty}} \approx\left\|\sup _{Q \ni \cdot} \frac{1}{|Q|^{1+\beta / n}} \int_{Q}\left|h-h_{Q}\right|\right\|_{p}
$$

The proof of the first part of (a) may be found in [3], pages 14 and 38, the proof of the second part of (a) in [8], and the proof of (b) in [11].

Fix a cube $Q=Q\left(x_{Q}, s\right)$, and $x \in Q$. Fix $g \in L^{p}$ and let $g_{1}=g \chi_{2 Q}$, $g_{2}=g-g_{1}$. Observe that $\mathrm{C}_{f} g=\mathrm{C}_{\left(f-f_{Q}\right)} g$, and we have:

$$
\begin{aligned}
& \frac{1}{|Q|^{1+\beta / n}} \int_{Q}\left|\mathrm{C}_{f} g-\left(\mathrm{C}_{f} g\right)_{Q}\right| \\
&= \frac{1}{|Q|^{1+\beta / n}} \int_{Q}\left|\mathrm{C}_{\left(f-f_{Q}\right)} g-\left(\mathrm{C}_{\left(f-f_{Q}\right)} g\right)_{Q}\right| \\
& \leq \frac{2}{|Q|^{1+\beta / n}} \int_{Q}\left|\mathrm{C}_{\left(f-f_{Q}\right)} g-T\left(\left(f-f_{Q}\right) g_{2}\right)\left(x_{Q}\right)\right| \\
& \leq \frac{2}{|Q|^{1+\beta / n}} \int_{Q}\left|\left(f-f_{Q}\right) T g\right|+\frac{2}{|Q|^{1+\beta / n}} \int_{Q}\left|T\left(\left(f-f_{Q}\right) g_{1}\right)\right| \\
& \quad \quad+\frac{2}{|Q|^{\beta / n}} \sup _{y \in Q}\left|T\left(\left(f-f_{Q}\right) g_{2}\right)(y)-T\left(\left(f-f_{Q}\right) g_{2}\right)\left(x_{Q}\right)\right| \\
&=\mathrm{I}+\mathrm{II}+\mathrm{III} .
\end{aligned}
$$

We estimate these terms individually:

$$
\begin{aligned}
\frac{1}{|Q|^{1+\beta / n}} \int_{Q}\left|\left(f-f_{Q}\right) T g\right| & \leq \frac{1}{|Q|^{\beta / n}} \sup _{y \in Q}\left|f(y)-f_{Q}\right| \frac{1}{|Q|} \int_{Q}|T g| \\
& \leq C\|f\|_{\dot{\Lambda}_{\beta}} M(T g)(x)
\end{aligned}
$$

where we used Lemma 1.5(a). Thus, $I \leq C\|f\|_{\dot{\Lambda}_{\beta}} M(T g)(x)$.

To get II, we need the following modification of a lemma from [6]: 
Lemma 1.6. With the above notation, we have:

$$
\left\|\left(f-f_{Q}\right) g_{1}\right\|_{r} \leq C|Q|^{1 / r+\beta / n}\|f\|_{\dot{\Lambda}_{\beta}} M_{r}(g)(x)
$$

Proof.

$$
\begin{aligned}
\frac{1}{|Q|} \int_{\mathbf{R}^{n}}\left|f-f_{Q}\right|^{r}\left|g_{1}\right|^{r} & =C \frac{1}{|2 Q|} \int_{2 Q}\left|f-f_{Q}\right|^{r}|g|^{r} \\
& \leq C\left(\sup _{y \in 2 Q}\left|f(y)-f_{Q}\right|\right)^{r} M_{r}^{r}(g)(x) \\
& \leq C|Q|^{r \beta / n}\|f\|_{\dot{\Lambda}_{\beta}}^{r} M_{r}^{r}(g)(x) .
\end{aligned}
$$

Using the boundedness of $T$, together with the Hölder's inequality, we obtain

$$
\begin{aligned}
\frac{1}{|Q|^{1+\beta / n}} \int_{Q}\left|T\left(\left(f-f_{Q}\right) g_{1}\right)\right| & \leq \frac{1}{|Q|^{1+\beta / n}}\left\|T\left(\left(f-f_{Q}\right) g_{1}\right)\right\|_{r} \cdot|Q|^{1-1 / r} \\
& \leq C|Q|^{-\beta / n-1 / r}\left\|\left(f-f_{Q}\right) g_{1}\right\|_{r} \\
& \leq C\|f\|_{\dot{\Lambda}_{\beta}} M_{r}(g)(x) .
\end{aligned}
$$

Thus, II $\leq C\|f\|_{\dot{\Lambda}_{\beta}} M_{r}(g)(x)$.

III: We need the following well known fact (see, e.g., [3], p. 14). Let $Q^{*} \subset Q$. Then

$$
\left|f_{Q^{*}}-f_{Q}\right| \leq C\|f\|_{\dot{\Lambda}_{\beta}}|Q|^{\beta / n} .
$$

We now estimate III.

$$
\begin{aligned}
& \left|T\left(\left(f-f_{Q}\right) g_{2}\right)(y)-T\left(\left(f-f_{Q}\right) g_{2}\right)\left(x_{Q}\right)\right| \\
= & \left|\int_{\mathbf{R}^{n}}\left(K(y-z)-K\left(x_{Q}-z\right)\right)\left(f(z)-f_{Q}\right) g_{2}(z) d z\right| \\
\leq & C \int_{(2 Q)^{c}} \frac{\left|y-x_{Q}\right|}{\left|x_{Q}-z\right|^{n+1}}\left|f(z)-f_{Q}\right||g(z)| d z \\
\leq & C \sum_{k=2}^{\infty} \int_{2^{k} Q \backslash 2^{k-1} Q} 2^{-k}\left|2^{k} Q\right|^{-1}\left(\left|f(z)-f_{2^{k} Q}\right|+\left|f_{2^{k} Q}-f_{Q}\right|\right)|g(z)| d z \\
\leq & C \sum_{k=2}^{\infty} 2^{-k}\left|2^{k} Q\right|^{\beta / n}\|f\|_{\dot{\Lambda}_{\beta}} M(g)(x)+C\|f\|_{\dot{\Lambda}_{\beta}} \sum_{k=2}^{\infty} 2^{-k}\left|2^{k} Q\right|^{\beta / n} M(g)(x) \\
\leq & C\|f\|_{\dot{\Lambda}_{\beta}}|Q|^{\beta / n} \sum_{k=2}^{\infty} 2^{-k+\beta k} M(g)(x) \\
= & C\|f\|_{\dot{\Lambda}_{\beta}}|Q|^{\beta / n} M(g)(x) .
\end{aligned}
$$


Thus, III $\leq C\|f\|_{\dot{\Lambda}_{\beta}} M(g)(x)$.

Putting these estimates together, we obtain

$$
\frac{1}{|Q|^{1+\beta / n}} \int_{Q}\left|\mathrm{C}_{f} g-\left(\mathrm{C}_{f} g\right)_{Q}\right| \leq C\|f\|_{\dot{\Lambda}_{\beta}}\left(M(T g)(x)+M_{r}(g)(x)+M(g)(x)\right) \text {. }
$$

We now take the supremum over all $Q$ such that $x \in Q$, and $L^{p}$ the norm of both sides, and using Lemma $1.5(\mathrm{~b})$ we conclude that

$$
\left\|\mathrm{C}_{f} g\right\|_{\dot{F}_{p}^{\beta, \infty}} \leq C\|f\|_{\dot{\Lambda}_{\beta}}\|g\|_{p}
$$

Thus, $(\mathrm{a}) \Rightarrow(\mathrm{b})$ is proved.

(b) $\Rightarrow(\mathrm{a})$ : We are going to show, that there exist $s, t, 1<s, t<\infty$, such that $1 / s-1 / t=\beta / n$, and $\mathrm{C}_{f}$ is bounded from $L^{s}\left(\mathbf{R}^{n}\right)$ to $L^{t}\left(\mathbf{R}^{n}\right)$, and $\left\|\mathrm{C}_{f}\right\|_{L^{s} \rightarrow L^{t}} \leq C\left\|\mathrm{C}_{f}\right\|_{L^{p} \rightarrow \dot{F}_{q}^{\beta, \infty}}$.

Claim 1.7. There exists $\beta_{0}, 0 \leq \beta_{0} \leq \beta$ such that $1 / p-\left(\beta-\beta_{0}\right) / n>0$ and $\beta_{0} / n+1 / p<1$.

Proof. For $0 \leq z \leq \beta, z \mapsto 1 / p-(\beta-z) / n$ is increasing and, if $z=\beta$, it is positive. Thus, there are two possibilities:

(a) for $z=0,1 / p-(\beta-z) / n=1 / p-\beta / n>0$, in which case we let $\beta_{0}=0$,

(b) there exists $z_{0}, 0 \leq z_{0}<\beta$ such that $1 / p-\left(\beta-z_{0}\right) / n=0$. Then, $z_{0} / n+$

$1 / p=\beta / n<1$. Then, there exists $\beta_{0}>z_{0}$ such that $\beta_{0} / n+1 / p<1$, and

$1 / p-\left(\beta-\beta_{0}\right) / n>1 / p-\left(\beta-z_{0}\right) / n=0$.

The claim is proved.

Claim 1.7 gives us a $\beta_{0}$. If this $\beta_{0}=0$, then $1 / p-\beta / n>0$. Choose $1<q<\infty$ such that $1 / p-1 / q=\beta / n$. Then, by Sobolev-Besov embedding,

$$
\left\|\mathrm{C}_{f} g\right\|_{q}=\left\|\mathrm{C}_{f} g\right\|_{\dot{F}_{q}^{02}} \leq C\left\|\mathrm{C}_{f} g\right\|_{\dot{F}_{p}^{\beta \infty}} \leq C\left\|\mathrm{C}_{f}\right\|_{L^{p} \rightarrow \dot{F}_{q}^{\beta, \infty}}\|g\|_{p} .
$$

Thus, we obtained the pair $s, t(p, q)$ indicated above Claim 1.7. If $\beta_{0}>0$, choose $1<r<\infty$ such that $1 / p-1 / r=\left(\beta-\beta_{0}\right) / n$, and $1<q<\infty$ such that $1 / q-1 / p=\beta_{0} / n$. Both of these choices are possible by the properties of $\beta_{0}$. We use the Sobolev-Besov embedding twice, and obtain:

$$
\left\|\mathrm{C}_{f} g\right\|_{\dot{F}_{r}^{\beta_{0}, 2}} \leq C\left\|\mathrm{C}_{f} g\right\|_{\dot{F}_{p}^{\beta, \infty}} \leq C\left\|\mathrm{C}_{f}\right\|_{L^{p} \rightarrow \dot{F}_{p}^{\beta, \infty}}\|g\|_{p} \leq C\left\|\mathrm{C}_{f}\right\|_{L^{p} \rightarrow \dot{F}_{p}^{\beta, \infty}}\|g\|_{\dot{F}_{q}^{\beta_{0}, 2}},
$$

so, $\mathrm{C}_{f}: \dot{F}_{q}^{\beta_{0}, 2} \rightarrow \dot{F}_{r}^{\beta_{0}, 2}$ is bounded. By duality (see [4]) we get that $\mathrm{C}_{f}^{*}$ : $\dot{F}_{r^{\prime}}^{-\beta_{0}, 2} \rightarrow \dot{F}_{q^{\prime}}^{-\beta_{0}, 2}$ is bounded. Observe that $\mathrm{C}_{f}^{*}=-\mathrm{C}_{\bar{f}}$, so, we have that $\mathrm{C}_{f}$ : 
$\dot{F}_{r^{\prime}}^{-\beta_{0}, 2} \rightarrow \dot{F}_{q^{\prime}}^{-\beta_{0}, 2}$ is bounded. By interpolation (see, again [4]), we get that $\mathrm{C}_{f}: \dot{F}_{s}^{0,2} \rightarrow \dot{F}_{t}^{0,2}$ is bounded, with $1 / s=\left(1 / q+1 / r^{\prime}\right) / 2=(1+\beta / n) / 2,1 / t=$ $\left(1 / q^{\prime}+1 / r\right) / 2=(1-\beta / n) / 2$. Thus, we get $\mathrm{C}_{f}: L^{s} \rightarrow L^{t}$ is bounded, with $1 / s-1 / t=\beta / n$.

Now, it follows that $f \in \dot{\Lambda}_{\beta}$ (see [6], Example 2), and $\|f\|_{\dot{\Lambda}_{\beta}} \leq C\left\|\mathrm{C}_{f}\right\|_{L^{p} \rightarrow \dot{F}_{p}^{\beta, \infty}}$.

Finally, the equivalence of (a) and (c) was proved in [6] (Example 2).

We now turn to the proof of Theorem 1.4:

Proof of Theorem 1.4. (a) $\Rightarrow$ (b): Fix $g \in L^{p}\left(\mathbf{R}^{n}\right)$ and recall that $x_{Q}$ denotes the center of a cube $Q$, and $g_{1}=g \chi_{2 Q}, g_{2}=g-g_{1}$.

$$
\begin{gathered}
\quad\left\|\mathrm{C}_{f}^{\alpha} g\right\|_{\dot{F}_{q}^{\beta, \infty}} \leq C\left\|\sup _{Q \ni \cdot \frac{1}{|Q|^{1+\beta / n}}} \int_{Q}\left|\mathrm{C}_{f}^{\alpha} g-\left(\mathrm{C}_{f}^{\alpha} g\right)_{Q}\right|\right\|_{q} \\
=C\left\|\sup _{Q \ni \cdot} \frac{1}{|Q|^{1+\beta / n}} \int_{Q}\left|\mathrm{C}_{\left(f-f_{Q}\right)}^{\alpha} g-\left(\mathrm{C}_{\left(f-f_{Q}\right)}^{\alpha} g\right)_{Q}\right|\right\|_{Q} \\
\leq C\left\|\sup _{Q \ni \cdot} \frac{1}{|Q|^{1+\beta / n}} \int_{Q}\left|\mathrm{C}_{\left(f-f_{Q}\right)}^{\alpha} g-I^{\alpha}\left(\left(f-f_{Q}\right) g_{2}\right)\left(x_{Q}\right)\right|\right\|_{q} \\
\leq C\left\|\sup _{Q \ni \cdot|Q|^{1+\beta / n}} \int_{Q}\left|\left(f-f_{Q}\right) I^{\alpha} g\right|\right\|_{q} \\
\quad+C\left\|\sup _{Q \ni \cdot} \frac{1}{|Q|^{1+(\alpha+\beta) / n}} \int_{Q}\left|I^{\alpha}\left(\left(f-f_{Q}\right) g\right)-I^{\alpha}\left(\left(f-f_{Q}\right) g_{2}\right)\left(x_{Q}\right)\right|\right\|_{p} .
\end{gathered}
$$

The last inequality follows from the following lemma, with $\beta$ in the place of $\gamma$.

Lemma 1.8 ([3], pp. 71-72). Let $1<p<q<\infty, 1 / p-1 / q=\alpha / n$. Suppose for each cube $Q$ we have a function $h^{Q}$, defined on this cube. Then, for $0 \leq \gamma$ :

$$
\left\|\sup _{Q \ni} \frac{1}{|Q|^{1+\gamma / n}} \int_{Q}\left|h^{Q}\right|\right\|_{q} \leq C\left\|\sup _{Q \ni \cdot} \frac{1}{|Q|^{1+\gamma / n+\alpha / n}} \int_{Q}\left|h^{Q}\right|\right\|_{p},
$$

where the constant $C$ depends only on $p, q, \alpha$ and $n$.

This lemma, as proved in [3], specifies that the functions $h^{Q}$ have the form $h-h_{Q}$, but the proof carries over immediately to the more general case above. 
Thus,

$$
\begin{aligned}
& \quad\left\|\mathrm{C}_{f}^{\alpha} g\right\|_{\dot{F}_{q}^{\beta, \infty}} \\
& \leq C\left\|\sup _{Q \ni \cdot} \frac{1}{|Q|^{1+\beta / n}} \int_{Q}\left|\left(f-f_{Q}\right) I^{\alpha} g\right|\right\|_{q} \\
& \quad+C\left\|\sup _{Q \ni \cdot} \frac{1}{|Q|^{1+(\alpha+\beta) / n}} \int_{Q}\left|I^{\alpha}\left(\left(f-f_{Q}\right) g_{1}\right)\right|\right\|_{p} \\
& \quad+C\left\|\sup _{Q \ni \cdot \mid} \frac{1}{|Q|^{(\alpha+\beta) / n}} \sup _{y \in Q}\left|I^{\alpha}\left(\left(f-f_{Q}\right) g_{2}\right)(y)-I^{\alpha}\left(\left(f-f_{Q}\right) g_{2}\right)\left(x_{Q}\right)\right|\right\|_{p} \\
& =\mathrm{I}+\mathrm{II}+\mathrm{III} .
\end{aligned}
$$

Now, we make a temporary assumption that $\alpha+\beta<1$, and estimate each part separately, as in the previous proof.

I: For each $x \in Q$ we get by Lemma $1.5(\mathrm{a})$,

$$
\begin{aligned}
\frac{1}{|Q|^{\beta / n}} \frac{1}{|Q|} \int_{Q}\left|\left(f-f_{Q}\right) I^{\alpha} g\right| & \leq \frac{1}{|Q|^{\beta / n}}\left(\sup _{y \in Q}\left|f(y)-f_{Q}\right|\right)\left(\frac{1}{|Q|} \int_{Q}\left|I^{\alpha} g\right|\right) \\
& \leq C\|f\|_{\dot{\Lambda}_{\beta}} M\left(I^{\alpha} g\right)(x),
\end{aligned}
$$

so $I \leq C\|f\|_{\dot{\Lambda}_{\beta}}\left\|I^{\alpha} g\right\|_{q} \leq C\|f\|_{\dot{\Lambda}_{\beta}}\|g\|_{p}$.

II: Choose $r, 1<r<p$, and $\bar{r}$ such that $(1 / r)-(1 / \bar{r})=\alpha / n$. Such $\bar{r}$ exists, since $r<p<n / \alpha$. We proceed as in the previous proof,

$$
\begin{aligned}
\frac{1}{|Q|^{1+(\alpha+\beta) / n}} \int_{Q}\left|I^{\alpha}\left(\left(f-f_{Q}\right) g_{1}\right)\right| & \leq \frac{1}{|Q|^{1+(\alpha+\beta) / n}}\left\|I^{\alpha}\left(\left(f-f_{Q}\right) g_{1}\right)\right\|_{\bar{r}}|Q|^{1 / \bar{r}^{\prime}} \\
& \leq C|Q|^{-1-\alpha / n-\beta / n+1-1 / \bar{r}}\left\|\left(f-f_{Q}\right) g_{1}\right\|_{r} \\
& =C|Q|^{-1 / r-\beta / n}\left\|\left(f-f_{Q}\right) g_{1}\right\|_{r} \\
& \leq C\|f\|_{\dot{\Lambda}_{\beta}} M_{r}(g)(x),
\end{aligned}
$$

by Lemma 1.6. Thus, II $\leq C\|f\|_{\dot{\Lambda}_{\beta}}\left\|M_{r}(g)\right\|_{p} \leq C\|f\|_{\dot{\Lambda}_{\beta}}\|g\|_{p}$. 
III: We proceed as in the previous proof. Let $y \in Q$,

$$
\begin{aligned}
& \frac{1}{|Q|^{(\alpha+\beta) / n}}\left|I^{\alpha}\left(\left(f-f_{Q}\right) g_{2}\right)(y)-I^{\alpha}\left(\left(f-f_{Q}\right) g_{2}\right)\left(x_{Q}\right)\right| \\
\leq & \frac{C}{|Q|^{(\alpha+\beta) / n}} \int_{(2 Q)^{c}} \frac{\left|y-x_{Q}\right|}{\left|x_{Q}-z\right|^{n+1-\alpha}}\left|f(z)-f_{Q}\right| \cdot|g(z)| d z \\
\leq & \frac{C}{|Q|^{(\alpha+\beta) / n}} \sum_{k=2}^{\infty} \int_{2^{k} Q \backslash 2^{k-1} Q} 2^{-k}\left|2^{k} Q\right|^{-1+\alpha / n}\left|f(z)-f_{Q}\right| \cdot|g(z)| d z \\
\leq & C \sum_{k=2}^{\infty} 2^{-k+k \alpha+k \beta} \frac{1}{\left|2^{k} Q\right|^{\beta / n}} \cdot \frac{1}{\left|2^{k} Q\right|} \int_{2^{k} Q}\left|f(z)-f_{2^{k} Q}\right| \cdot|g(z)| d z \\
\leq & C\|f\|_{\dot{\Lambda}_{\beta}} M(g)(x) .
\end{aligned}
$$

So, III $\leq C\|f\|_{\dot{\Lambda}_{\beta}}\|g\|_{p}$. Thus,

$$
\left\|\mathrm{C}_{f}^{\alpha} g\right\|_{\dot{F}_{q}^{\beta, \infty}} \leq C\|f\|_{\dot{\Lambda}_{\beta}}\|g\|_{p}
$$

Now, we get rid of the assumption $\alpha+\beta<1$. For this, we need the following easy observation:

Fact 1.9. There exist $m, k_{0} \geq 0$ integers, and $\delta>0$ such that $\alpha=$ $\delta+m \beta / 2^{k_{0}}$, with $\beta+\delta<1$.

Now, observe that since $\beta+\beta / 2^{k_{0}}<1$, the part of the theorem already proved says that if $1 / u-1 / w=\beta_{0} / n$, then $\mathrm{C}_{f}^{\beta_{0}}: L^{u} \rightarrow \dot{F}_{w}^{\beta, \infty}$ is bounded, with $\beta_{0}=\beta / 2^{k_{0}}, 1<u, w<\infty$.

Fact 1.10. Suppose $C_{f}^{\gamma}: L^{u} \rightarrow \dot{F}_{w}^{\beta, \infty}$ is bounded for every $u, w, 1 / u-$ $1 / w=\gamma / n, 1<u, w<\infty, 0<\gamma<n-\beta_{0}$. Then $C_{f}^{\gamma+\beta_{0}}: L^{u} \rightarrow \dot{F}_{v}^{\beta, \infty}$ is bounded for every $u, v, 1 / u-1 / v=\left(\gamma+\beta_{0}\right) / n, 1<u, v<\infty$.

Proof. Choose $u<n /\left(\gamma+\beta_{0}\right), g \in L^{u}$.

$$
\begin{aligned}
\mathrm{C}_{f}^{\gamma+\beta_{0}} g & =I^{\gamma} I^{\beta_{0}}(f g)-f I^{\gamma} I^{\beta_{0}} g \\
& =I^{\gamma}\left(I^{\beta_{0}} f g-f I^{\beta_{0}} g\right)+I^{\gamma} f I^{\beta_{0}} g-f I^{\gamma} I^{\beta_{0}} g \\
& =I^{\gamma}\left(\mathrm{C}_{f}^{\beta_{0}} g\right)+\mathrm{C}_{f}^{\gamma}\left(I^{\beta_{0}} g\right) \\
& =A g+B g .
\end{aligned}
$$


(A) $\mathrm{C}_{f}^{\beta_{0}}: L^{u} \rightarrow \dot{F}_{r}^{\beta, \infty} ; 1 / u-1 / r=\beta_{0} / n$, so $A: L^{u} \rightarrow \dot{F}_{r}^{\beta+\gamma, \infty} \hookrightarrow \dot{F}_{v}^{\beta, \infty}$, where $1 / r-1 / v=\gamma / n$ and thus $1 / u-1 / v=\left(\gamma+\beta_{0}\right) / n$.

(B) $I^{\beta_{0}}: L^{u} \rightarrow \dot{F}_{u}^{\beta_{0}, 2} \hookrightarrow L^{s} ; 1 / u-1 / s=\beta_{0} / n$, so $B: L^{u} \rightarrow \dot{F}_{v}^{\beta, \infty}$, where $1 / s-1 / v=\gamma / n$ and thus $1 / u-1 / v=\left(\beta_{0}+\gamma\right) / n$. The embeddings used are the Sobolev-Besov embeddings ([5]).

Now, the hypothesis of Fact 1.10 is satisfied for $\gamma=\delta$ (since $\beta+\delta<1$ ). We then apply Fact $1.10 m$ times to increase this $\gamma$ by $\beta_{0}, m$ times. Thus, by induction, (a) $\Rightarrow(\mathrm{b})$ is proved for $\delta+m \beta_{0}=\alpha$. Observe that we also get that $\left\|\mathrm{C}_{f}^{\alpha}\right\|_{L^{p} \rightarrow \dot{F}_{q}^{\beta, \infty}} \leq C\|f\|_{\dot{\Lambda}_{\beta}}$.

(b) $\Rightarrow$ (a): Choose a $\mathrm{C}^{\infty}$ function $a$ with the following properties:

$$
\begin{aligned}
& \operatorname{supp} a \subset Q(0,3), \quad \int_{\mathbf{R}^{n}} a=0 \\
& |a(x)| \leq 1, \quad\left|\frac{\partial}{\partial x_{i}} a(x)\right| \leq 1, \quad i=1, \ldots, n,
\end{aligned}
$$

$a$ is radial, and there exists $x \notin B(0,1)$ with $a(x) \neq 0$.

Here, $B(0,1)$ is the ball centered at 0 with radius 1 . It follows that $a$ is an atom for the cube $Q(0,1)$ for the space $\dot{F}_{q^{\prime}}^{-\beta, 1}$, and $\|a\|_{F_{q^{\prime}}^{-\beta, 1}}=1$ (see [13], pp. 17-18). We know that $\left(\dot{F}_{q^{\prime}}^{-\beta, 1}\right)^{*}=\dot{F}_{q}^{\beta, \infty}$ (see [4], p. 60). Define:

$$
\nu(x)=\int_{\mathbf{R}^{n}}|y-x|^{\alpha-n}\left(\chi_{B(0,1)}(x) a(y)-a(x) \chi_{B(0,1)}(y)\right) d y .
$$

Let $\nu_{t}(x)=1 / t^{n} \nu(x / t)$. By change of variables, we get

$$
f * \nu_{t}\left(x_{0}\right)=\omega_{\alpha}^{-1} t^{-n-\alpha} \int_{\mathbf{R}^{n}} \mathrm{C}_{f}^{\alpha}\left(\chi_{B\left(x_{0}, t\right)}\right)(x) a^{\prime}(x) d x,
$$

where $a^{\prime}(x)=a\left(\left(x-x_{0}\right) / t\right)$. So, for $x_{0} \in \mathbf{R}^{n}$,

$$
\begin{aligned}
\left|f * \nu_{t}\left(x_{0}\right)\right| & \leq C t^{-n-\alpha}\left\|a^{\prime}\right\|_{\dot{F}_{q^{\prime}}^{-\beta, 1}}\left\|\mathrm{C}_{f}^{\alpha}\left(\chi_{B\left(x_{0}, t\right)}\right)\right\|_{\dot{F}_{q}^{\beta, \infty}} \\
& \leq C t^{-n-\alpha} t^{\beta+n / q^{\prime}}\left\|\mathrm{C}_{f}^{\alpha}\right\|_{L^{p} \rightarrow \dot{F}_{q}^{\beta, \infty}} \cdot t^{n / p} \\
& =C\left\|\mathrm{C}_{f}^{\alpha}\right\|_{L^{p} \rightarrow \dot{F}_{q}^{\beta, \infty}} \cdot t^{\beta} .
\end{aligned}
$$

We used the homogeneity properties of the spaces $L^{p}$ and $\dot{F}_{q^{\prime}}^{-\beta, 1}$ :

$$
\left\|a^{\prime}\right\|_{\dot{F}_{q^{\prime}}^{-\beta, 1}}=t^{\beta+n / q^{\prime}}\|a\|_{\dot{F}_{q^{\prime}}^{-\beta, 1}},
$$

and

$$
\left\|\chi_{B\left(x_{0}, t\right)}\right\|_{p}=t^{n / p}\left\|\chi_{B(0,1)}\right\|_{p} .
$$

Let $\hat{\nu}$ be the Fourier transform of $\nu$. 
Lemma 1.11. $\hat{\nu}$ has the Tauberian property; that is, for every $\xi \in \mathbf{R}^{n}$, $\xi \neq 0$, there exists $t>0$ such that $\hat{\nu}(t \xi) \neq 0$.

Proof. Observe that $\nu$ is radial, and thus $\hat{\nu}$ is radial. So, if there exists $\xi$ such that for every $t>0, \hat{\nu}(t \xi)=0$, we would have $\hat{\nu} \equiv 0$, and thus $\nu \equiv 0$. By assumption, there exists an open set $U$ with $B(0,1) \cap U=\varnothing$, and $a(x) \neq 0$ for $x \in U$. On the other hand, if $x \notin B(0,1)$,

$$
\nu(x)=\int_{\mathbf{R}^{n}}|y-x|^{\alpha-n}\left(-a(x) \chi_{B(0,1)}(y)\right) d y=-a(x) \int_{B(0,1)}|y-x|^{\alpha-n} d y .
$$

Thus, $\nu(x) \neq 0$ for $x \in U$.

Thus, $\hat{\nu}$ has the Tauberian property, and by (1.1),

$$
\sup _{t>0} t^{-\beta}\left\|f * \nu_{t}\right\|_{\infty}<C\left\|\mathrm{C}_{f}^{\alpha}\right\|_{L^{p} \rightarrow \dot{F}_{q}^{\beta, \infty}} .
$$

Using Calderón's reproducing formula due to Janson and Taibleson ([7]), we get

$$
f=f_{0}+P
$$

with $f_{0} \in \dot{\Lambda}_{\beta},\left\|f_{0}\right\|_{\dot{\Lambda}_{\beta}} \leq C\left\|\mathrm{C}_{f}^{\alpha}\right\|_{L^{p} \rightarrow \dot{F}_{q}^{\beta, \infty}}$ and $P$ a polynomial. From part (a) $\Rightarrow$ (b) in this proof, we get that $\mathrm{C}_{f_{0}}^{\alpha}: L^{p} \rightarrow \dot{F}_{q}^{\beta, \infty}$ is bounded, and thus $\mathrm{C}_{P}^{\alpha}: L^{p} \rightarrow$ $\dot{F}_{q}^{\beta, \infty}$ is bounded. Let $P(x)=\sum_{|\gamma| \leq M} a_{\gamma} x^{\gamma}$. We will show that $P$ is actually a constant. Fix $a$, a smooth atom for the space $\dot{F}_{q^{\prime}}^{-\beta, 1}, x_{0} \in \mathbf{R}^{n}, s>0$.

$$
\begin{aligned}
& \left\langle\mathrm{C}_{P}^{\alpha} \chi_{Q\left(t x_{0}, t s\right)}, a(\cdot / t)\right\rangle \\
= & C \int_{\mathbf{R}^{n}} \int_{\mathbf{R}^{n}}(P(y)-P(x))|y-x|^{\alpha-n} \chi_{Q\left(x_{0}, s\right)}\left(\frac{y}{t}\right) a\left(\frac{x}{t}\right) d y d x \\
= & C \sum_{i=0}^{M} \sum_{|\gamma|=i} a_{\gamma} \int_{\mathbf{R}^{n}} \int_{\mathbf{R}^{n}}\left(y^{\gamma}-x^{\gamma}\right)|y-x|^{\alpha-n} \chi_{Q\left(x_{0}, s\right)}\left(\frac{y}{t}\right) a\left(\frac{x}{t}\right) d y d x \\
= & C t^{n+\alpha} \sum_{i=0}^{M} t^{i} \sum_{|\gamma|=i} a_{\gamma} \int_{\mathbf{R}^{n}} \int_{\mathbf{R}^{n}}\left(y^{\gamma}-x^{\gamma}\right)|y-x|^{\alpha-n} \chi_{Q\left(x_{0}, s\right)}(y) a(x) d y d x \\
= & C t^{n+\alpha} \sum_{i=0}^{M} t^{i} d_{i} .
\end{aligned}
$$

On the other hand,

$$
\left|\left\langle\mathrm{C}_{P}^{\alpha} \chi_{Q\left(t x_{0}, t s\right)}, a\left(\frac{\cdot}{t}\right)\right\rangle\right| \leq\left\|\mathrm{C}_{P}^{\alpha}\right\|_{L^{p} \rightarrow \dot{F}_{q}^{\beta, \infty}}\left\|\chi_{Q\left(t x_{0}, t s\right)}\right\|_{p}\left\|a\left(\frac{\dot{t}}{t}\right)\right\|_{\dot{F}_{q^{\prime}}^{-\beta, 1}} \leq C t^{\alpha+\beta+n}
$$


$\left(s, x_{0}\right.$ fixed). Thus, $\left|\sum_{i=0}^{M} t^{i} d_{i}\right| \leq C t^{\beta}$ with $0<\beta<1$, so $d_{i} \equiv 0$ for every $i$. In particular,

$$
\left\langle\mathrm{C}_{P_{i}}^{\alpha} \chi_{Q\left(x_{0}, s\right)}, a\right\rangle=0
$$

for every $i$, where $P_{i}$ is the homogeneous part of $P$ of degree $i$. Thus,

$$
\left\langle\mathrm{C}_{P_{i}}^{\alpha} g, h\right\rangle=0 \quad \forall g \in L^{p}, h \in \dot{F}_{q^{\prime}}^{-\beta, 1} .
$$

This means that $\left\|\mathrm{C}_{P_{i}}^{\alpha} g\right\|_{\dot{F}_{q}^{\beta, \infty}}=0$, so $\mathrm{C}_{P_{i}}^{\alpha} g$ is a constant a.e. for every $g \in L^{p}\left(\mathbf{R}^{n}\right)$. Let $i>0$, and suppose $A \subset\{x: 1<|x|<2\}$. It is then easy to observe that $\mathrm{C}_{P_{i}}^{\alpha} \chi_{A}$ is continuous on the complement of $\left\{x:\left(\frac{1}{2}\right)<|x|<3\right\}$. Thus, $\mathrm{C}_{P_{i}}^{\alpha} \chi_{A}$ is constant on this set. Let $A=\left\{x: P_{i}(x)>0\right\} \cap\{x: 1<|x|<2\}$, and $B=\left\{x: P_{i}(x)<0\right\} \cap\{x: 1<|x|<2\}$. Since $i>0$, we have $P_{i}(0)=0$.

$$
\begin{aligned}
& \mathrm{C}_{P_{i}}^{\alpha} \chi_{A}(0)=C \int_{A} P_{i}(y)|y|^{\alpha-n} d y \geq 0, \\
& \mathrm{C}_{P_{i}}^{\alpha} \chi_{B}(0)=C \int_{B} P_{i}(y)|y|^{\alpha-n} d y \leq 0 .
\end{aligned}
$$

Suppose $x_{1} \in A, x_{2} \in B$.

$$
\begin{aligned}
\mathrm{C}_{P_{i}}^{\alpha} \chi_{A}\left(t x_{1}\right) & =C \int_{A} P_{i}(y)\left|y-t x_{1}\right|^{\alpha-n} d y-C P_{i}\left(t x_{1}\right) \int_{A}\left|y-t x_{1}\right|^{\alpha-n} d y, \\
\mathrm{C}_{P_{i}}^{\alpha} \chi_{B}\left(t x_{2}\right) & =C \int_{B} P_{i}(y)\left|y-t x_{2}\right|^{\alpha-n} d y-C P_{i}\left(t x_{2}\right) \int_{B}\left|y-t x_{2}\right|^{\alpha-n} d y .
\end{aligned}
$$

If $t>3$, both (1.4) and (1.5) become independent of $t$, and thus, both (1.4) and (1.5) have limits as $t \rightarrow \infty$, and these limits are equal to (1.2) and (1.3) respectively. In (1.4) and (1.5), the first terms tend to 0 as $t \rightarrow \infty$, and the second terms are negative and positive respectively. Thus, the limit of (1.4) is $\leq 0$ and the limit of (1.5) is $\geq 0$. Comparing this with (1.2) and (1.3), we get that $(1.2)=(1.3)=0$. Thus, both $A$ and $B$ have measure 0 , and being open sets, are empty. So, $P_{i}(x) \equiv 0$ on $\{x: 1<|x|<2\}$, and being a polynomial, $P_{i} \equiv 0$. Thus, polynomial $P$ is a constant, and therefore $P \in \dot{\Lambda}_{\beta}$, and thus finally $f \in \dot{\Lambda}_{\beta}$.

$$
\begin{aligned}
& \text { (a) } \Rightarrow(\mathrm{c}) \text { : By definition, if } f \in \dot{\Lambda}_{\beta} \text {, then }|f(y)-f(x)| \leq\|f\|_{\dot{\Lambda}_{\beta}}|y-x|^{\beta} \text {. So, } \\
& \left|\mathrm{C}_{f}^{\alpha} g(x)\right| \leq C\|f\|_{\dot{\Lambda}_{\beta}} \int_{\mathbf{R}^{n}}|y-x|^{\alpha+\beta-n}|g(y)| d y=C\|f\|_{\dot{\Lambda}_{\beta}} I^{\alpha+\beta}(|g|)(x),
\end{aligned}
$$

and $I^{\alpha+\beta}: L^{p} \rightarrow L^{r}$ is bounded, provided $1 / p-1 / r=(\alpha+\beta) / n$. 
(c) $\Rightarrow$ (a): We follow the method in [6]. Choose $z_{0} \in \mathbf{R}^{n}$ such that in $Q\left(z_{0}, 2\right),|x|^{n-\alpha}$ can be written as the absolutely convergent Fourier series, $|x|^{n-\alpha}=\sum_{m=0}^{\infty} a_{m} e^{i\left\langle v_{m}, x\right\rangle}$, with $\sum\left|a_{m}\right|<\infty$. Choose $x_{0} \in \mathbf{R}^{n}, t>0$, and let $Q=Q\left(x_{0}, t\right), Q^{z_{0}}=Q\left(x_{0}+z_{0} t, t\right)$.

$$
\begin{aligned}
& \frac{1}{|Q|} \frac{1}{\mid Q^{z_{0} \mid}} \int_{Q}\left|\int_{Q^{z_{0}}}(f(y)-f(x)) d y\right| d x \\
= & \frac{1}{t^{2 n}} \int_{Q} s(x)\left(\int_{Q^{z_{0}}}(f(y)-f(x))|y-x|^{\alpha-n}|y-x|^{n-\alpha} d y\right) d x
\end{aligned}
$$

where $\left.s(x)=\overline{\operatorname{sgn}\left(\int_{Q^{z_{0}}}(f(y)-f(x)) d y\right.}\right)$. For $x \in Q, y \in Q^{z_{0}}$ we have $(y-x) / t \in$ $Q\left(z_{0}, 2\right)$, so this expression is equal to

$$
\begin{aligned}
& \frac{t^{n-\alpha}}{t^{2 n}} \int_{Q} s(x)\left(\int_{Q^{z_{0}}}(f(y)-f(x))|y-x|^{\alpha-n}\left|\frac{y-x}{t}\right|^{n-\alpha} d y\right) d x \\
= & t^{-n-\alpha} \sum_{m=0}^{\infty} a_{m} \int_{Q} s(x)\left(\int_{Q^{z_{0}}}(f(y)-f(x))|y-x|^{\alpha-n} e^{i\left\langle v_{m}, y / t\right\rangle} d y\right) e^{-i\left\langle v_{m}, x / t\right\rangle} d x \\
= & C t^{-n-\alpha}\left|\sum_{m=0}^{\infty} a_{m} \int_{\mathbf{R}^{n}} \mathrm{C}_{f}^{\alpha}\left(\chi_{Q^{z_{0}}} e^{i\left\langle v_{m}, \cdot / t\right\rangle}\right)(x) \chi_{Q}(x) e^{-i\left\langle v_{m}, x / t\right\rangle} s(x) d x\right| \\
\leq & C t^{-n-\alpha} \sum_{m=0}^{\infty}\left|a_{m}\right|\left\|\mathrm{C}_{f}^{\alpha}\left(\chi_{Q^{z_{0}}} e^{i\left\langle v_{m}, \cdot / t\right\rangle}\right)\right\|_{r} \cdot\left\|\chi_{Q}\right\|_{r^{\prime}} \\
\leq & C t^{-n-\alpha}\left\|\mathrm{C}_{f}^{\alpha}\right\|_{L^{p} \rightarrow L^{r}} t^{n / p} t^{n-n / r} \sum_{m=0}^{\infty}\left|a_{m}\right| \\
= & C t^{\beta}\left\|\mathrm{C}_{f}^{\alpha}\right\|_{L^{p} \rightarrow L^{r} .}
\end{aligned}
$$

We conclude that

$$
\frac{1}{|Q|^{1+\beta / n}} \int_{Q}\left|f-f_{Q}\right| \leq \frac{2}{|Q|^{1+\beta / n}} \int_{Q}\left|f-f_{Q^{z_{0}}}\right| \leq C\left\|\mathrm{C}_{f}^{\alpha}\right\|_{L^{p} \rightarrow L^{r}} .
$$

From Lemma 1.5 (a) we conclude that $f \in \dot{\Lambda}_{\beta}$, and $\|f\|_{\dot{\Lambda}_{\beta}} \leq C\left\|\mathrm{C}_{f}^{\alpha}\right\|$. Theorem 1.4 is proved.

2. Generalized commutator operator. Suppose $0<\beta<k \leq n, k$ an integer (in particular, $k=[\beta]+1$ ), and $n$ the dimension of the ambient space. Let us define

$$
\begin{aligned}
& \mathrm{C}_{f, k} g(x)=\int_{\mathbf{R}^{n}} \Delta_{h / k}^{k} f(x) K(h) g(x+h) d h, \\
& \mathrm{C}_{f, k}^{\alpha} g(x)=\int_{\mathbf{R}^{n}} \Delta_{h / k}^{k} f(x) K^{\alpha}(h) g(x+h) d h .
\end{aligned}
$$

We have the following two theorems, which generalize the theorems from the previous section, where we considered the particular case $k=1$. 
Theorem 2.1. Let $1<p<q<\infty ;(1 / p)-(1 / q)=\beta / n$. Under the assumptions above, the following are equivalent:

(a) $f=f_{1}+P$, with $f_{1} \in \dot{\Lambda}_{\beta}$ and $P$ a polynomial of degree less than $k$.

(b) $\mathrm{C}_{f, k}: L^{p} \rightarrow L^{q}$ is a bounded operator.

Theorem 2.2. Let $1<p<q<\infty ;(1 / p)-(1 / q)=(\alpha+\beta) / n$. Under the assumptions above, the following are equivalent:

(a) $f=f_{1}+P$, with $f_{1} \in \dot{\Lambda}_{\beta}$ and $P$ a polynomial of degree less than $k$.

(b) $\mathrm{C}_{f, k}^{\alpha}: L^{p} \rightarrow L^{q}$ is a bounded operator.

If, in particular, $k=[\beta]+1$, then (a) in both theorems says that $f \in \dot{\Lambda}_{\beta}$.

Proof of Theorem 2.1. (a) $\Rightarrow$ (b): If the degree of $P$ is less than $k$, then $\Delta_{h}^{k} P=0$. Thus, we need to show that if $f \in \dot{\Lambda}_{\beta}$, then $\mathrm{C}_{f, k}$ is bounded from $L^{p}$ to $L^{q}$. If $f \in \dot{\Lambda}_{\beta}$, then $\left|\Delta_{h}^{k} f(x)\right| \leq\|f\|_{\dot{\Lambda}_{\beta}} \cdot|h|^{\beta}$, so

$$
\left|\mathrm{C}_{f, k} g(x)\right| \leq C\|f\|_{\dot{\Lambda}_{\beta}} \int_{\mathbf{R}^{n}} \frac{|h|^{\beta}}{|h|^{n}}|g(x+h)| d h=C\|f\|_{\dot{\Lambda}_{\beta}} I^{\beta}(|g|)(x) .
$$

Since $I^{\beta}$ is bounded from $L^{p}$ to $L^{q}$, (b) follows.

(b) $\Rightarrow$ (a): We are going to use the characterization of the Besov spaces from [10].

Theorem $2.3([10])$. Let $0<\beta<k$, with $k$ an integer. Suppose $f \in$ $\mathcal{S}^{\prime} \cap L_{\text {loc }}^{1}$. The following are then equivalent:

(a) $f=f_{1}+P$, where $f_{1} \in \dot{B}_{\infty}^{\beta, \infty}$ and $P$ is a polynomial of degree less than $k$.

(b) There exists $z_{0} \in \mathbf{R}^{n}$ such that

$$
\sup _{t>0} t^{-\beta} \sup _{x_{0} \in \mathbf{R}^{n}} \frac{1}{|Q|} \frac{1}{\mid Q^{z_{0} \mid}}\left(\int_{Q}\left|\int_{Q^{z_{0}}}\left(\Delta_{(y-x) / k}^{k} f(x)\right) d y\right| d x\right) \leq C<\infty
$$

where $Q=Q\left(x_{0}, t\right)$, and $Q^{z_{0}}=Q\left(x_{0}+z_{0} t, t\right)$.

If these conditions hold, then $\left\|f_{1}\right\|_{\dot{B}_{\infty}^{\beta, \infty}}$ is comparable with the best possible $C$ in (b). 
We are going to show that condition (b) of Theorem 2.3 holds. We follow the proof of the last part of Theorem 1.4. We choose $z_{0}$ such that $1 / K(x)=$ $\sum_{m=0}^{\infty} a_{m} e^{i\left\langle v_{m}, x\right\rangle}$ with $\sum\left|a_{m}\right|<\infty$, if $x \in Q\left(z_{0}, 2\right)$. We choose $x_{0} \in \mathbf{R}^{n}, t>0$, and we let $Q=Q\left(x_{0}, t\right), Q^{z_{0}}=Q\left(x_{0}+z_{0} t, t\right)$. Then,

$$
\begin{aligned}
& \left|\frac{1}{|Q|} \frac{1}{\mid Q^{z_{0} \mid}} \int_{Q} \int_{Q^{z_{0}}}\left(\Delta_{y-x}^{k} f(x)\right) d y d x\right| \\
= & \frac{1}{t^{2 n}}\left|\int_{Q} \int_{Q^{z_{0}}}\left[\Delta_{y-x}^{k} f(x)\right] K(y-x) \frac{1}{K(y-x)} d y d x\right| \\
= & t^{-n} \mid \sum_{m=0}^{\infty} a_{m} \int_{\mathbf{R}^{n}}\left(\int_{\mathbf{R}^{n}}\left(\Delta_{y-x}^{k} f(x)\right) K(y-x) \chi_{Q^{z_{0}}}(y) e^{i\left\langle v_{m}, y\right\rangle / t} d y\right) \\
& \times \chi_{Q}(x) e^{-i\left\langle v_{m}, x\right\rangle / t} d x \mid \\
\leq & t^{-n} \sum_{m=0}^{\infty}\left|a_{m}\right|\left\|\mathrm{C}_{f, k}\left(\chi_{Q^{z_{0}}} \cdot e^{i\left\langle v_{m}, \cdot\right\rangle / t}\right)\right\|_{q}\left\|\chi_{Q}\right\|_{q^{\prime}} \\
\leq & t^{-n}\left\|\mathrm{C}_{f, k}\right\|_{L^{p} \rightarrow L^{q}}\left|Q^{z_{0}}\right|^{1 / p}|Q|^{1-1 / q} \sum_{m=0}^{\infty}\left|a_{m}\right| \\
= & C\left\|\mathrm{C}_{f, k}\right\|_{L^{p} \rightarrow L^{q}} t^{\beta} .
\end{aligned}
$$

Thus, condition (b) of Theorem 2.3 holds.

The proof of Theorem 2.2 is identical.

\section{REFERENCES}

[1] S. Chanillo, A note on commutators, Indiana Univ. Math. J. 31 (1982), 7-16.

[2] R. R. Coifman, R. Rochberg $\mathcal{E}$ G. Weiss, Factorization theorems for Hardy spaces in several variables, Ann. of Math. 103 (1976), 611-635.

[3] R. A. DeVore \& R. C. Sharpley, Maximal functions measuring smoothness, Mem. Amer. Math. Soc. 47 (1984).

[4] M. Frazier 83 B. Jawerth, A discrete transform and decompositions of distribution spaces, J. Funct. Anal. 93 (1990), 34-170.

[5] M. Frazier, B. Jawerth \& G. Weiss, Littlewood-Paley Theory and the Study of Function Spaces, CBMS-AMS Regional Conf. Series 79 (1991).

[6] S. JAnson, Mean oscillation and commutators of singular integral operators, Ark. Math. 16 (1978), 263-270.

[7] S. Janson $\&$ M. Taibleson, I teoremi di rappresentazione di Calderón, Rend. Sem. Mat. Univ. Politec. Torino 39 (1981), 27-35.

[8] S. Janson, M. Taibleson \& G. Weiss, Elementary characterization of the Morrey-Campanato spaces, Lect. Notes in Math. 992 (1983), 101-114.

[9] M. Paluszynski, Characterization of Lipschitz Spaces Via the Commutator Operator of Coifman, Rochberg and Weiss; A Multiplier Theorem For the Semigroup of Contractions, Ph.D. Thesis, Washington University, St. Louis, 1992. 
[10] M. Paluszynski \& M. Taibleson, Characterization of the Besov spaces, Proceedings, Miraflores 1992 (to appear).

[11] A. Seeger, A Note on Triebel-Lizorkin spaces, Approximation and Function Spaces, PWN, Warsaw, 1989.

[12] E. Stein, Singular Integrals and Differentiability Properties of Functions, Princeton University Press, Princeton, NJ, 1970.

[13] R. ToRres, Boundedness results for operators with singular kernels on distribution spaces, Mem. Amer. Math. Soc. 90 (1991).

[14] A. Uchiyama, On the compactness of operators of Hankel type, Tôhoku Math. J. 30 (1978), 163-171.

Mathematical Institute

University of Wrocław

Plac Grunwaldzki 2/4

50-384 Wrocław, Poland

E-mail: mpal@math.uni.wroc.pl

Received: October 19, 1993. 